Unfallchirurg 2018 $121: 858-859$

https://doi.org/10.1007/s00113-018-0551-2

(c) Springer Medizin Verlag GmbH, ein Teil von Springer Nature 2018

CrossMark

\title{
Christian Krettek
}

Medizinische Hochschule Hannover (MHH), Hannover, Deutschland

\section{Motorisierte intramedulläre Verlängerungsnägel}

Die Distraktionsosteogenese ist seit vielen Jahrzehnten ein bewährtes und sicheres Konzept zur Behandlung von Extremitätendeformitäten [6, 7]. In der Kombination mit internen (Nagel/Platte) Implantaten konnte die Zeit mit oft störender und in einigen Aspekten nachteiliger externer Fixation erheblich reduziert werden $[10,11]$.

Motorisierte, intramedulläre Implantate stehen seit etlichen Jahren v.a. zur Knochenverlängerung zur Verfügung. Die Antriebsenergie wurde dabei mechanisch über einen Ratschenmechanismus (beim Gang oder mit definierten Rotationsbewegungen [2, 5]) oder elektrisch (Batterie, Kabel, Induktion [1]) dem Antriebsaggregat zugeführt.

Jetzt steht eine neue Generation von motorisierten intramedullären Implantaten zur Verfügung, die den Zugang und die Anwendung interner Knochendistraktion weitervereinfacht hat [4]. Wesentliches Kennzeichen dieser Implantate ist ein Magnetantrieb, der Energie und Steuersignale von außen durch Weichteile und Knochen über Induktion erhält. Das Konzept war ursprünglich für die bariatrische Chirurgie zur steuerbaren Reduktion der Magenpassage von Scott Pool und Blair Walker entwickelt worden und wurde 2006 patentiert. Die erste Implantation am Knochen zur Verlängerung erfolgte 2011 in Neuseeland. Mechanische Schwächen der ersten Generation (P1) wurden in einer überarbeiteten Version (P2) im Jahre 2013 adressiert.

Das ermöglicht nun in deutlich breiterem Maße neue Ansätze nicht nur für die lineare Extremitätenverlängerung, sondern auch für die Therapie von komplexen kombinierten Rekonstruktionsproblemen bei angeborenen oder erworbenen, oft posttraumatischen Fehlstellun- gen. Das hat sich auch in den Implantationszahlen niedergeschlagen. Wurden im Jahre 2017 noch 1027 Implantate eingesetzt, sind es bis August 2018 weltweit insgesamt bereits über 8000 .

In dieser Ausgabe habe ich einige dieser Techniken für verschiedene Anwendungsbereiche und Patientengruppen ohne Anspruch auf Vollständigkeit zusammengetragen. So enthält das vorliegende Leitthema Beiträge zur Anwendung von motorisierten Nägeln zur Extremitätenverlängerung bei Kindern [3] und zur Anwendung am Humerus [12].

Große Probleme in der Unfallchirurgie bestehen aber v.a. bei der Behandlung von Knochendefekten. Hier besteht großer Bedarf nach einfachen geschlossenen Verfahren für einen komplett inneren Segmenttransport. Diese Problematik ist von der Industrie weitgehend und lange ungehört geblieben, mit dem Argument eines nur kleinen „Nischenmarktes“.

Nun sollten sich aber Unfallchirurgen trotz zunehmender Beschränkung durch z. T. sinnleere Vorschriften des Medizinproduktegesetzes und trotz Entwicklungsagonie vieler Hersteller und deren assoziierten „Kommissionen“ nicht davon abhalten kreativ, innovativ und v.a. schnell nach Lösungen zu suchen.

Dass dies mit Fantasie und Hartnäckigkeit auch ohne die Entwicklungsabteilungen der Industrie und hochbürokratisierte institutionalisierte Forschungsförderung gelingen kann, zeigen die Beiträge mit den Lösungen „plate assisted bone segment transport" (PABST) [8] und Magic Tube [9].

Hier wurde mit wenig Aufwand, einfachen Mitteln, kreativen Ansätzen und schneller Umsetzung gezeigt, wie Lö- 
sungs- und Implantatweiterentwicklung auch sein kann.

Das Verfahren "plate assisted bone segment transport“ (PABST) erlaubt es erstmals, mit einem Knochenverlängerungsnagel und einer zusätzlichen Platte Knochensegmentdefekte über Kallusdistraktion aufzufüllen.

Mit dem MagicTube (Modulares Assistenz Gerät für Intersegmentale Corticalisdefekte mittels Tubus Erweiterung) ist es weltweit zum ersten Mal gelungen, Knochensegmentdefekte mit kommerziell erhältlichen Knochenverlängerungsnägeln und einem einfachen Zusatzmodul komplett über eine Kallusdistraktion aufzufüllen und den Knochen bei Bedarf (meist liegt auch eine Verkürzung vor) optional ohne weiteren zusätzlichen Eingriff zu verlängern. Von Herstellerseite werden bald ebenfalls Implantate zur Verfügung stehen, die speziell für den Knochensegmenttransport entwickelt wurden. Die damit zu erwartende weitere Zunahme an Anwendungen setzt entsprechendes Wissen und Fertigkeiten voraus. Als Herausgeber von Der Unfallchirurg sehen wir uns in der Verpflichtung, unsere Leser diesbezüglich auf dem neuesten Stand $\mathrm{zu}$ halten und weiter $\mathrm{zu}$ informieren.

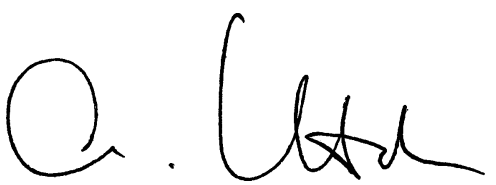

Prof. Dr. Christian. Krettek, FRACS, FRCSEd

\section{Korrespondenzadresse}

Prof. Dr. Christian Krettek,
FRACS, FRCSEd
Medizinische Hochschule
Hannover (MHH)
Carl-Neuberg-Str. 1,
30625 Hannover,
Deutschland
Krettek.Christian@
mh-hannover.de

Interessenkonflikt. C. Krettek gibt an, dass kein Interessenkonflikt besteht.

\section{Literatur}

1. Baumgart R, Betz A, Schweiberer L (1997) A fully implantable motorized intramedullary nail for limb lengthening and bone transport. Clin Orthop RelatRes 343(343):135-143

2. Cole JD, Justin D, Kasparis T, DeVlught D, Knobloch C (2001) The intramedullary skeletal kinetic distractor (ISKD): first clinical results of a new intramedullary nail for lengthening of the femur and tibia. Injury 32(Suppl4):SD129-39

3. Frommer A, Rödl R, Gosheger G, Vogt B (2018) Anwendung motorisierter Verlängerungsmarknäge am wachsenden Skelett - Indikationsspektrum und Limitationen. Unfallchirurg. https://doi.org/ 10.1007/s00113-018-0541-4

4. Green SA, Herzenberg JE, Standard SCN (2012) PRECICE intramedullary limb lengthening system. Surgical Technique Guide. Tibial operative technique. Ellipse Technologies, Inc 13900 Alton Parkway, Suite 123, Irvine, CA 92618 USA

5. Guichet JM, Deromedis B, Donnan LT, Peretti G, Lascombes P, Bado F (2003) Gradual femoral lengthening with the Albizzia intramedullary nail JBone Jt Surg Am 85-A (5:838-848

6. Ilizarov GA (1989) The Tension-Stress Effect on the Genesis and Growth of Tissues: Part I. The Influence of Stability of Fixation and Soft-Tissue Preservation. Clin Orthop Relat Res 238:249-281

7. Ilizarov GA (1989) The tension-stress effect on the genesis and growth of tissues: Part II. The influence of the rate and frequency of distraction. Clin Orthop Relat Res 239:263-285

8. Kähler UO (2018) Plate Assisted Segmental Bone Transport (PABST) mit einem motorisiertem intramedullären Verlängerungsnagel und Platte - Eine neue Technik zur Behandlung von Knochensegmentdefekten in Tibia und Femur. Unfallchirurg. https://doi.org/10.1007/s00113018-0546-z

9. Krettek C (2018) MagicTube: Neue Möglichkeiten zum komplett internen Knochensegmenttransport und optionaler Verlängerung - Ein neues Zusatzmodul für motorisierte Verlängerungsnägel zur Behandlung von großen Knochendefekten. Unfallchirurg. https://doi.org/10.1007/s00113018-0549-9

10. Oh CW, Apivatthakakul T, Oh JK, Kim JW, Lee HJ, Kyung HS, et al (2013) Bone transport with an external fixator and a locking plate for segmental tibial defects. The bone \& Joint Journal 95B(12):1667-1672.

11. Raschke MJ, Mann JW, Oedekoven G, Claudi BF (1992) Segmental transport after unreamed intramedullary nailing. Preliminary report of a „Monorail" System. Clin Orthop Relat Res 282:233-240

12. Wozasek GE, ZakL (2018) Intramedulläre Oberarmverlängerung. Der Unfallchirurg. https://doi.org/ 10.1007/s00113-018-0542-3

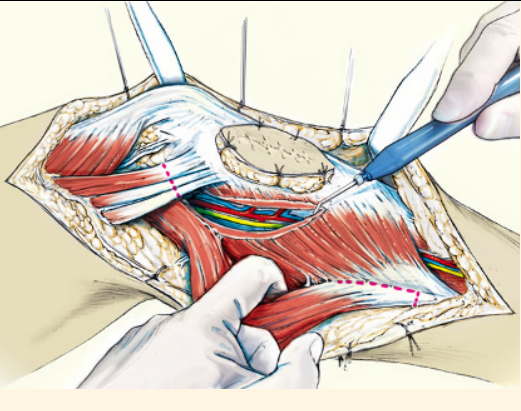

Suchen Sie noch mehr zum Leitthema?

Die Zeitschrift Operative Orthopädie und Traumatologie bietet Ihnen fundierte Informationen zu bewährten und neuen Operationstechniken der Orthopädie, Unfall-, Hand- und Wiederherstellungschirurgie. In den aufwendig illustrierten Beiträgen werden die Operationsverfahren Schritt für Schritt vorgestellt.

Passend zum Leitthema "Motorisierte intramedulläre Verlängerungsnägel“ in dieser Ausgabe können Sie sich z.B. in der OOT über folgende Operationstechniken informieren:

- Wachstumsprothesen zur

Rekonstruktion von Defekten der unteren Extremitäten beim Kind Schinhan M, Funovics P, Dominkus M et al. (2012) Operat Orthop Traumatol 24:235-246

- Verlängerung der unteren Extremität mit dem Intramedullary Skeletal Kinetic Distractor (ISKD) Hankemeier S, Gösling T, Pape HC et al. (2005) Orthop Traumatol 17:79-101

- Daumenrekonstruktion durch kontinuierliche Verlängerung des ersten Mittelhandknochens Preisser P, Rudolf K, Partecke BD (1997) Oper Orthop Traumatol 9:150-161

Mit e.Med - den maßgeschneiderten Fortbildungsabos von Springer Medizin - haben Sie Zugriff auf alle Inhalte von SpringerMedizin.de. Sie können schnell und komfortabel in den Zeitschriften Ihres Fachgebietss recherchieren und auf alle Inhalte im Volltext zugreifen.

Weitere Infos zu e.Med finden Sie auf springermedizin.de unter "Abos" 